\title{
Bowel, Urinary, and Sexual Function in Patients with Low to Intermediate Risk Prostate Cancer on Active Surveillance and Primary Focal Cryotherapy
}

\author{
Jordan Mendelson ${ }^{1^{*}}$ (D), Jessica Sommer ${ }^{2}$, Meredith Akerman, $\mathrm{MS}^{3}$ and Aaron Katz, MD ${ }^{1,2}$
}

${ }^{1}$ New York University Long Island School of Medicine, USA

${ }^{2}$ Department of Urology, New York University Langone Hospital-Long Island, USA

${ }^{3}$ Biostatistics Core, Division of Health and Research, New York University Langone Hospital-Long Island, USA

\begin{abstract}
Objectives: Both active surveillance (AS) and primary focal cryotherapy (PFC) are considered suitable approaches for men with low to intermediate risk prostate cancer (PCa). We aim to identify any differences in bowel, urinary, and sexual function that may exist between patients on AS and those who have undergone PFC.

Methods: A retrospective review of the 1,230 patients in the IRB-approved Prostate Cancer Database at NYU Langone Hospital-Long Island was performed. 320 of the patients were either on AS or had received PFC at time of review. Exclusion criteria included incomplete questionnaires and additional salvage therapy following PFC. Data from the 179 remaining patients were reviewed for demographics, treatment (AS vs. PFC), prostate-specific antigen (PSA) levels at time of treatment, Gleason scores and grade groups, time to clinical follow-up, and responses to both the Expanded Prostate Cancer Index Composite (EPIC) and the International Index of Erectile Function (IIEF) questionnaires. Descriptive and comparative statistics were used to compare survey outcomes by treatment method.
\end{abstract}

Results: Of the 179 patients with low to intermediate risk prostate cancer 118 (65.9\%) were on AS and 61 (34.1\%) received PFC. The two groups varied slightly in pre-treatment Gleason scores and grade groups, with higher-risk patients in the PFC group ( $<0.001)$. There was no difference in median age (68 vs. 71 years, $p=0.107$ ) or the use of phosphodiesterase- 5 inhibitors such as sildenafil or tadalafil ( $24 \%$ vs. $30 \%, p=0.425)$ between the AS and PFC groups at the time of survey completion. The median time from start date of AS or date of PFC to date of survey completion was 38 months and 43 months, respectively $(p=0.586)$. There was no difference between the two groups in either bowel or urinary function ( $p=0.947$ and $p=0.278$, respectively). The PFC group had higher rates of use of both alpha-blockers and 5 - $\alpha$-reductase inhibitors ( $p<0.001$ for both). Overall subjective analysis of sexual function was worse among the PFC group ( $p$ 0.001). $20.3 \%$ of patients on AS and $27.9 \%$ in the PFC group denied any sexual stimulation over four weeks prior to survey completion. The differences in rates of ejaculation and sensation of orgasm with sexual stimulation between the two groups were not significant ( $p=0.061$ and $p=0.351$, respectively). The difference in satisfaction with overall sex life between the two groups was also not significant $(p=0.086)$.

Conclusions: PFC for low to intermediate risk prostate cancer does not impair bowel or urinary function to a significant degree when compared to AS. Though it impairs subjective analysis of overall sexual function, it does not significantly alter rates of ejaculation or sensation of orgasm, or satisfaction with one's sex life.

\section{Keywords}

Quality of life, Cryotherapy, Active surveillance, Prostate cancer

\section{Abbreviations}

PCa: Prostate Cancer; PSA: Prostate-Specific Antigen; PFC: Primary Focal Cryotherapy; AS: Active Surveillance; EPIC: Expanded Prostate Cancer Index Composite; IIEF: International Index of Erectile Function; IQR: Interquartile Range

\section{Introduction}

Prostate cancer (PCa) is the most common cause of cancer and the fifth most common cause of cancer death among men worldwide [1]. The D'Amico classification model stratifies men with PCa into groups with low, intermediate, or high risk of biochemical recurrence after treatment according to the patients' clinical TNM stage, Gleason score, and pretreatment prostate-specific antigen (PSA) level [2]. While some authors have proposed changes to the D'Amico model, 
Citation: Mendelson J, Sommer J, Akerman M, et al. (2021) Bowel, Urinary, and Sexual Function in Patients with Low to Intermediate Risk Prostate Cancer on Active Surveillance and Primary Focal Cryotherapy. Ann Minim Invasive Surg 3(1):24-30

it is still widely used to risk stratify men with PCa [3]. For patients with low to intermediate risk $\mathrm{PCa}$, several approaches such as prostatectomy and radiotherapy have historically been considered suitable $[4,5]$. However, these procedures are often can be associated with relatively high risks of bowel, urinary, and sexual dysfunction [6]. More recently, evidence for the efficacy of focal, partial-gland treatment options for localized PCa has been steadily growing. Newer treatment options include focal cryotherapy, high-intensity focused ultrasound, irreversible electroporation, photodynamic therapy, and focused laser ablation [7]. These focal therapies offer the benefit of minimized lifetime treatment-related morbidity while still proving efficacious $[8,9]$. Primary focal cryotherapy (PFC), in particular, has proven to be effective in treating men with low to intermediate risk $\mathrm{PCa}$ in both shortterm and mid-term studies [10-13]. Cryotherapy refers to the controlled freezing of the prostate gland. The rapid decrease in temperature within the gland results in denaturation of proteins, disruption of blood supply, and apoptosis of cells upon thawing of the gland $[14,15]$.

In addition to these various treatment options, active surveillance (AS) is also considered a suitable approach for men with low or favorable intermediate risk localized PCa [16]. AS involves delivering curative treatment on recognition of disease progression via prostate biopsy and is intended to avoid invasive treatments and treatment-related morbidity in patients who do not necessarily require definitive treatment $[17,18]$. While individual cohorts and urologic organizations offer competing AS protocols, most suggest periodic digital rectal examination and PSA testing in addition to a confirmatory, repeat prostatic biopsy [19-27]. One modeling study found that AS prolongs life expectancy when compared to watchful waiting, which entails the administration of non-curative androgen deprivation therapy to patients on recognition of signs or symptoms of disease progression [28].

While both focal therapy and AS are considered appropriate options for men with low to intermediate risk PCa, these approaches are not necessarily equal due to the potential effect of treatment-related morbidity on patients' quality of life. In this study, we attempt to identify any differences in bowel, urinary, and sexual function that may exist between patients on AS and those who have undergone PFC. We believe we have a unique ability to examine differences in AS and PFC due to our extensive database which includes a large patient population encompassing both cohorts.

\section{Materials and Methods}

We conducted a retrospective review of the 1,230 patients in the IRB-approved Prostate Cancer Database at New York University Langone Hospital-Long Island. Of the patients in the database, 320 had been risk stratified as low to intermediate risk of biochemical recurrence and were either on AS or had received $P F C$ at the time of review. Each patient within the PFC cohort was treated with hemi-ablation of the prostate. Exclusion from the study included incomplete questionnaires at the time of review and additional salvage therapy following PFC. Figure 1 illustrates the patient selection criteria and process. Data from the 179 remaining patients were reviewed for demographics, treatment method (AS vs. PFC), PSA levels at time of initial treatment, Gleason scores and grade groups, time to clinical follow-up, responses to both the Expanded Prostate Cancer Index Composite (EPIC) and the International Index of Erectile Function (IIEF) questionnaires, and use of phosphodiesterase- 5 inhibitors, alpha blockers, and 5 - $\alpha$-reductase inhibitors. These patients initiated AS or had undergone PFC as early as 2006. The questionnaires were distributed to patients every three months during the first year after enrollment and every six months in each year thereafter. Both the EPIC and IIEF questionnaires have been validated and proven to be reliable in assessing the quality of life of patients with PCa $[29,30]$. The most recent subjective patient symptom scores in each of the EPIC domains (i.e., urinary function, bowel habits, sexual function) and IIEF domains (i.e., erectile function, orgasmic function, sexual desire, satisfaction with intercourse, and overall sexual satisfaction) were quantified using a six-point Likert scale. We then utilized both descriptive (e.g., median and IQR or frequency and percent) and comparative (e.g., MannWhitney test, chi-square test, Fisher's exact test) statistics to compare these quantified responses to the questionnaires by treatment method and subsequently calculated subdomain scores for bowel, urinary, and sexual function. Analyses were performed using SAS version 9.4. A result was considered statistically significant at $p<0.05$.

\section{Results}

Of the 179 included patients with low to intermediate risk $\mathrm{PCa}, 118$ (65.9\%) were on AS and 61 (34.1\%) received PFC. The PFC group had higher Gleason scores and Gleason grade groups at baseline $(p<0.001)$. There was no significant difference between the two groups in either race $(p=0.214)$ or ethnicity $(p=1.00)$. There was also no difference between the two groups in median age (68 vs. 71 years, $p=0.107$ ). The median time from initiation of AS or date of PFC to date of survey completion was 38 and 43 months, respectively $(p=0.586)$. Patient demographics and pre-treatment PCa characteristics are listed below in Table 1. Neither anxiety about post-treatment PSA levels nor fear of recurrence of PCa were significantly different between the two groups $(p=$ 0.494 and $p=0.551$, respectively).

There was no statistically significant difference between the AS and PFC groups in the bowel habit subdomain score $(p=0.947)$. The difference between the two groups in the urinary function subdomain score was also not statistically significant $(p=0.278)$. The AS and PFC groups did, however,

*Corresponding author: Jordan Mendelson, New York University Long Island School of Medicine, 101 Mineola Boulevard, NY 11501, USA, Tel: +1-(845)-709-4343

Accepted: August 16, 2021

Published online: August 18, 2021

Citation: Mendelson J, Sommer J, Akerman M, et al. (2021) Bowel, Urinary, and Sexual Function in Patients with Low to Intermediate Risk Prostate Cancer on Active Surveillance and Primary Focal Cryotherapy. Ann Minim Invasive Surg 3(1):24-30 
Citation: Mendelson J, Sommer J, Akerman M, et al. (2021) Bowel, Urinary, and Sexual Function in Patients with Low to Intermediate Risk Prostate Cancer on Active Surveillance and Primary Focal Cryotherapy. Ann Minim Invasive Surg 3(1):24-30

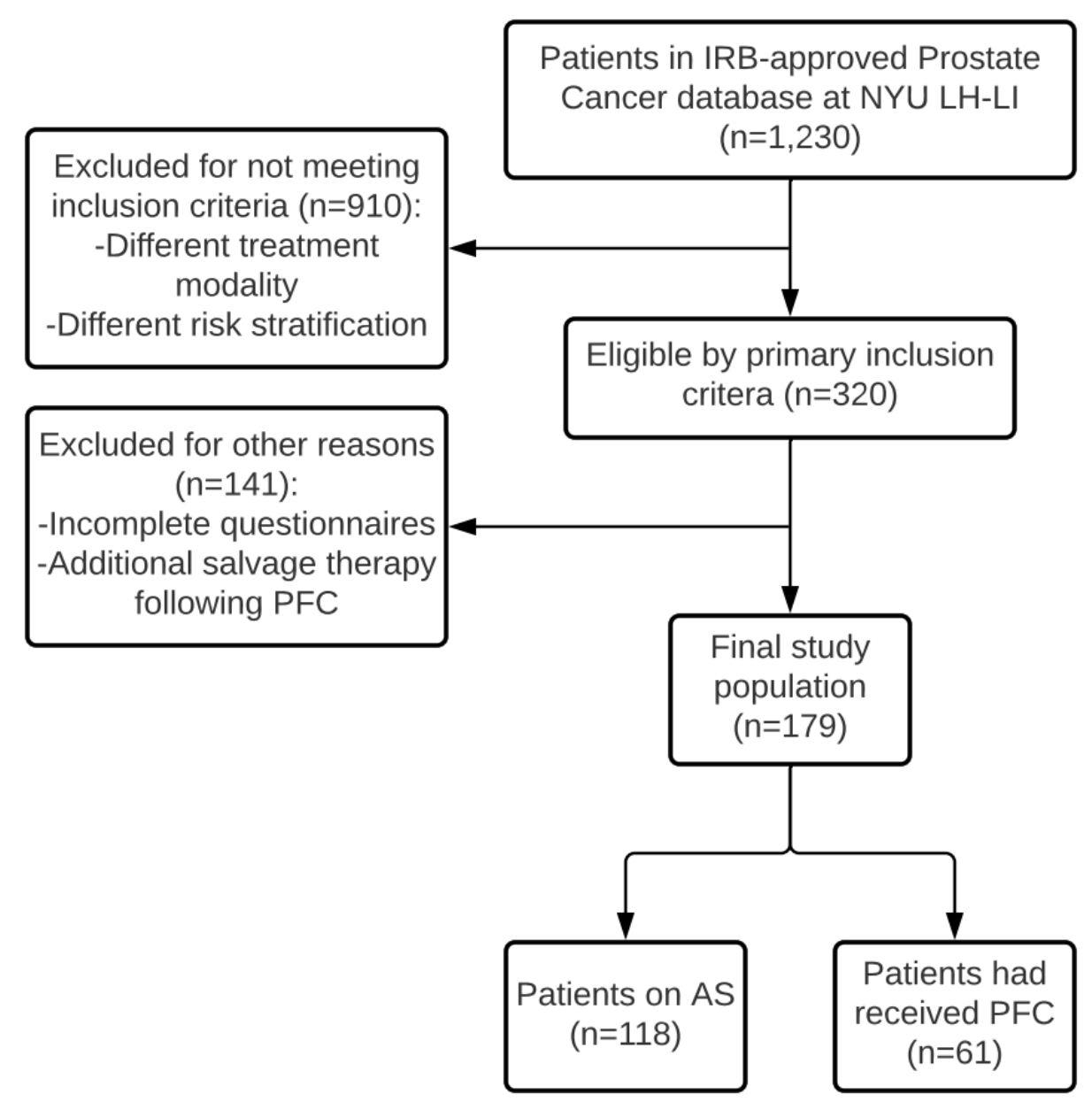

Figure 1: Consort diagram.

Table 1: Patient demographics and pre-treatment PCa characteristics.

\begin{tabular}{|l|l|l|l|}
\hline & Active Surveillance $(\mathbf{n = 1 1 8})$ & Primary Focal Cryotherapy $(\mathbf{n}=61)$ & Total (n= 179) \\
\hline Race $(p=0.214)$ & & & $2(1.1 \%)$ \\
\hline Asian & $0(0.0 \%)$ & $2(3.3 \%)$ & $8(4.5 \%)$ \\
\hline Black & $6(5.1 \%)$ & $2(3.3 \%)$ & $165(92.2 \%)$ \\
\hline White & $110(93.2 \%)$ & $55(90.2 \%)$ & $4(2.2 \%)$ \\
\hline Unknown/not reported & $2(1.7 \%)$ & $2(3.3 \%)$ & $5(2.8 \%)$ \\
\hline Ethnicity $p=1.00)$ & & & $161(89.9 \%)$ \\
\hline Hispanic/Latino & $3(2.5 \%)$ & $2(3.3 \%)$ & $13(7.3 \%)$ \\
\hline Not Hispanic/Latino & $106(89.8 \%)$ & $55(90.2 \%)$ & $69(65,74)$ \\
\hline Unknown/not reported & $9(7.6 \%)$ & $4(6.6 \%)$ & $71(66,74)$ \\
\hline Median Age ${ }^{*+}(p=0.107)$ & $68(64,74)$ & & $2(1.1 \%)$ \\
\hline Baseline Gleason Score ${ }^{\prime}(p<0.001)$ & & $0(0.0 \%)$ & $128(72.3 \%)$ \\
\hline Benign & $2(1.7 \%)$ & $32(53.3 \%)$ & $31(17.5 \%)$ \\
\hline$(3+3)=6$ & $96(82.0 \%)$ & $14(23.3 \%)$ & $11(6.2 \%)$ \\
\hline$(3+4)=7$ & $17(14.5 \%)$ & $9(15.0 \%)$ & $5(2.8 \%)$ \\
\hline$(4+3)=7$ & $2(1.7 \%)$ & $5(8.3 \%)$ & $5.4(3.8,6.7)$ \\
\hline$(4+4)=8$ & $0(0.0 \%)$ & $5.6(3.9,7.1)$ & \\
\hline Median Baseline PSA ${ }^{\#+}(p=0.566)$ & $5.3(3.8,6.7)$ & & \\
\hline
\end{tabular}

${ }^{*}$ At time of survey completion, "Most recent result prior to initiation of AS/treatment with PFC, ${ }^{+}$Median results provided with IQR. 
Citation: Mendelson J, Sommer J, Akerman M, et al. (2021) Bowel, Urinary, and Sexual Function in Patients with Low to Intermediate Risk Prostate Cancer on Active Surveillance and Primary Focal Cryotherapy. Ann Minim Invasive Surg 3(1):24-30

differ significantly in regard to their use of both alpha blockers (14.4\% vs. $42.6 \%, p<0.001)$ and 5 - $\alpha$-reductase inhibitors (5.9\% vs. $37.7 \%, p<0.001)$. Medication use is not being controlled for in this study -- this difference can be attributed to patient symptoms.

Overall subjective analysis of sexual function was worse among the PFC group ( $p<0.001)$. 24 patients on AS $(20.3 \%)$ and 17 patients in the PFC group (27.9\%) denied any sexual stimulation or intercourse over the four weeks prior to survey completion. After these 41 patients were excluded from analysis, there was no significant difference in the frequency with which patients in the two groups ejaculated in response to sexual intercourse or other sexual stimulation $(p=0.061)$. An additional two patients denied any sexual stimulation or intercourse over the four weeks prior to survey completion in response to the subsequent question regarding frequency of orgasm. After excluding these 43 patients from analysis, there was also no significant difference between the two groups in the frequency of the sensation of orgasm with sexual intercourse or other sexual stimulation $(p=0.351)$. There was no significant difference between the AS and PFC groups in satisfaction with overall sex life $(p=0.086)$. There was also no difference in the use of phosphodiesterase- 5 inhibitors such as sildenafil or tadalafil ( $24.3 \%$ vs. $30.2 \%, p=0.425)$ between the AS and PFC groups at the time of survey completion. Table 2 summarizes the results of the study below.

\section{Discussion}

While all treatment modalities utilized in treating men with PCa are associated with some treatment-related morbidity, these associated morbidities are not necessarily equal in frequency, duration, or severity. Recently, Marra, et al. examined long-term quality of life outcomes of AS and PFC [31]. The study intriguingly found that PFC led to fewer long-term complications than AS, though it did not yield significant oncological advantages compared to AS after ten years. Furthermore, it found that PFC prolonged time to both radical therapy and androgen deprivation therapy.

One systematic review of twelve studies revealed that patients who had undergone radiotherapy for localized PCa incurred higher rates of sexual dysfunction following treatment than those who had undergone focal cryotherapy [32]. Litwin, et al. found that radical prostatectomy and radiotherapy alike were associated with diminished urinary, bowel, and sexual function compared to patients' baseline [33]. Another study concluded that quality of life metrics in patients who had undergone cryotherapy were noninferior to those who had been treated with radical prostatectomy, radiotherapy, brachytherapy, or observation alone [34]. The CEASAR study demonstrated that bilateral nerve-sparing prostatectomy resulted in better sexual and urinary function than both unilateral nerve-sparing and non-nerve-sparing prostatectomy, though this difference was not significant in men with low baseline sexual function [35]. A large multicenter study found that, after five years of observation post-treatment, prostatectomy was associated with the worst urinary and sexual outcomes compared to active surveillance, radiotherapy, and brachytherapy [36]. All of these studies, in addition to many others, demonstrate the morbidity associated with definitive treatment for PCa.

Cryotherapy is an appropriate and effective treatment modality for low to intermediate risk PCa. Historically, cryotherapy was intended to treat the whole gland and thus often damaged the bilateral neurovascular plexuses posterolateral to the prostate. Focal cryotherapy involves hemi-ablation of the gland, consequently leaving the contralateral neurovascular plexus unaffected so continence and sexual function often remain intact [15]. A multicenter trial of whole gland cryotherapy for localized PCa revealed extensive morbidity associated with treatment, including impotence in $87 \%$ of previously potent men and incontinence in $8 \%$ of participants [37]. In contrast, an early pilot study of

Table 2: Summary of results.

\begin{tabular}{|l|l|l|l|}
\hline & Active Surveillance $\mathbf{( n = 1 1 8})$ & Primary Focal Cryotherapy (n= 61) & P-value \\
\hline Mean Bowel Function & 94.54 & 94.00 & 0.947 \\
\hline Mean Urinary Function & 83.76 & 86.60 & 0.278 \\
\hline Mean Sexual Function & 54.14 & 38.75 & $<0.001$ \\
\hline Mean Rate of Ejaculation & 4.17 & 3.68 & 0.061 \\
\hline Mean Rate of Sensation of Orgasm & 4.02 & 4.00 & 0.351 \\
\hline Mean Satisfaction with Overall Sex Life & 2.91 & 2.48 & 0.086 \\
\hline Use of Alpha Blockers & $17(14.4 \%)$ & $26(42.6 \%)$ & $<0.001$ \\
\hline Use of 5- $\alpha$-Reductase Inhibitors & $7(5.9 \%)$ & $23(37.7 \%)$ & $<0.001$ \\
\hline Use of PDE-5 Inhibitors & $27(24.3 \%)$ & $16(30.2 \%)$ & 0.425 \\
\hline Median Time to Survey Completion & 38 months & 43 months & 0.586 \\
\hline Anxiety About Post-Treatment PSA & 0.75 & 0.68 & 0.494 \\
\hline Fear of PCa Recurrence & 8.57 & 8.54 & 0.551 \\
\hline
\end{tabular}

$*_{n}=94$ for AS group, $n=44$ for PFC group, $\# n=95$ for AS group, $n=41$ for PFC group 
Citation: Mendelson J, Sommer J, Akerman M, et al. (2021) Bowel, Urinary, and Sexual Function in Patients with Low to Intermediate Risk Prostate Cancer on Active Surveillance and Primary Focal Cryotherapy. Ann Minim Invasive Surg 3(1):24-30

focal cryotherapy demonstrated preserved potency in seven of the nine study participants [38]. Additional studies have further illustrated the decreased morbidity associated with focal cryotherapy compared to whole gland cryotherapy $[10,14,15,39,40]$. Furthermore, some of these studies and others have demonstrated that loss of sexual function after focal cryotherapy is often transient in nature and its incidence may be decreasing $[14,32,34,41]$.

AS is often associated with greater quantified patientreported quality of life metrics than other approaches for men with PCa. A systematic review illustrated those men with localized PCa managed with AS have better or comparable quality of life metrics than those who had undergone radical treatments [42]. A similar study found that cryotherapy was associated with initially diminished bowel and sexual function compared to AS but noted that these deficits in function resolved over time [43].

The AS and PFC groups within this study did not differ in regards to most patient characteristics at baseline. Specifically, they did not differ significantly in race, ethnicity, or age. The PFC group did, however, have higher pretreatment Gleason scores and grade groups. This difference is likely intrinsic to this type of retrospective study, as patients with higher risk PCa are more likely to undergo definitive treatment. Additionally, although statistically significant, this difference in pre-treatment Gleason score can likely be attributed to just several patients within the PFC group with higher risk PCa. Similar to the results of various other studies, our analysis revealed a decline in subjective sexual function in men following treatment with cryotherapy, though it showed no significant difference in overall sexual satisfaction. Interestingly, the decline in sexual function observed in this study among patients in the PFC group seemingly did not return to baseline function over time, as determined by initial patient surveys; the median time from date of PFC to survey completion was 43 months, yet the observed decline in sexual function remained. The median time to follow-up in this study was 38 months for the AS group; this difference was not significant. Based on the available literature, these endpoints were far enough removed from treatment that sexual function would have been expected to return to baseline in the PFC group [14,32,34,41]. Urinary function and bowel function were not significantly impacted in the patients who had undergone PFC compared to the AS group, though the two groups did differ significantly in their use of both alpha blockers and 5 - $\alpha$-reductase inhibitors. The higher prevalence of medication use among the PFC group represents a potentially confounding variable in regard to the urinary function subdomain scores, as these classes of medications are both known to improve urinary function in certain patients.

This study was a single-center study. As such, more widespread investigation is merited in order to determine if these results are replicable in other patient populations. The retrospective design of this study also has inherent bias in patient selection. In addition, some of the PFC patients in this study were not enrolled in the IRB-approved Prostate Cancer Database at New York University Langone Hospital-
Long Island until many months or even years after receiving PFC. Since patients did not complete any validated surveys until their enrollment in the database, we cannot adequately assess changes in these patients' survey responses over time following treatment.

Despite these limitations, this study provides valuable insight into potential quality of life differences between patients on AS and those who have undergone PFC. This study adds to the limited existing literature on the topic by highlighting mid-term quality of life outcomes following AS and PFC, whereas the recent similar study by Marra, et al. examined long-term outcomes [31]. This study compared men who have undergone PFC for low to intermediate risk PCa with men on AS; because these two groups did not differ in patient characteristics at baseline, quality of life differences between the two groups measured at the study's endpoints can reasonably be attributed to the difference in treatment modality for the patients' low to intermediate risk PCa. As the validated surveys utilized in this study were distributed at set time points following treatment and were not retrospective, potential biases were minimized. As such, this study successfully quantifies differences in quality-of-life metrics that may occur following initiation of each of these treatment modalities. Still, additional systemic review comparing bowel, urinary, and sexual function among patients who received PFC versus other focal treatment modalities is indicated.

\section{Conclusions}

In men with low to intermediate risk PCa, PFC does not significantly impair bowel or urinary function when compared to AS. Though it does impair subjective analysis of overall sexual function, it notably does not significantly diminish rates of ejaculation or sensation of orgasm with sexual intercourse or other sexual stimulation, or the patients' satisfaction with their sex lives. PFC also does not significantly alter patients' anxiety about post-treatment PSA levels or fear of PCa recurrence when compared to AS.

\section{References}

1. Fitzmaurice C, Allen C, Barber RM, et al. (2017) Global, regional, and national cancer incidence, mortality, years of life lost, years lived with disability, and disability-adjusted life-years for 32 cancer groups, 1990 to 2015: A systematic analysis for the global burden of disease study global burden of disease cancer collaboration. JAMA Oncol 3: 524-548.

2. Hernandez DJ, Nielsen ME, Han M, et al. (2007) Contemporary evaluation of the D'Amico risk classification of prostate cancer. Urology 70: 931-935.

3. Rodrigues G, Warde P, Pickles T, et al. (2012) Pre-treatment risk stratification of prostate cancer patients: A critical review. Can Urol Assoc J 6: 121-127.

4. Bass EJ, Ahmed HU (2016) Focal therapy in prostate cancer: A review of seven common controversies. Cancer Treat Rev 51: 27-34.

5. Alicikus ZA, Yamada Y, Zhang Z, et al. (2011) Ten-year outcomes of high-dose, intensity-modulated radiotherapy for localized prostate cancer. Cancer 117: 1429-1437.

6. Wilt TJ, Ullman KE, Linskens EJ, et al. (2021) Therapies for 
Citation: Mendelson J, Sommer J, Akerman M, et al. (2021) Bowel, Urinary, and Sexual Function in Patients with Low to Intermediate Risk Prostate Cancer on Active Surveillance and Primary Focal Cryotherapy. Ann Minim Invasive Surg 3(1):24-30

clinically localized prostate cancer: A comparative effectiveness review. J Urol 20: 967-976.

7. Linares-Espinós E, Carneiro A, Martínez-Salamanca Jl, et al. (2018) New technologies and techniques for prostate cancer focal therapy. Minerva Urol Nefrol 70: 252-263.

8. Turpen R, Rosser CJ (2009) Focal therapy for prostate cancer: Revolution or evolution? BMC Urol 9: 2-2.

9. Ahmed HU, Freeman A, Kirkham A, et al. (2011) Focal therapy for localized prostate cancer: A phase I/II trial. J Urol 185: 12461254.

10. Bahn D, de Castro Abreu AL, Gill IS, et al. (2012) Focal cryotherapy for clinically unilateral, low-intermediate risk prostate cancer in 73 men with a median follow-up of 3.7 years. Eur Urol 62: 55-63.

11. Bahn DK, Silverman P, Lee F, et al. (2006) Focal prostate cryoablation: Initial results show cancer control and potency preservation. J Endourol 20: 688-692.

12. Onik G, Vaughan D, Lotenfoe R, et al. (2008) The "male lumpectomy": Focal therapy for prostate cancer using cryoablation results in 48 patients with at least 2-year follow-up. Urol Oncol 26: 500-505.

13. Truesdale MD, Cheetham PJ, Hruby GW, et al. (2010) An evaluation of patient selection criteria on predicting progressionfree survival after primary focal unilateral nerve-sparing cryoablation for prostate cancer: Recommendations for follow up. Cancer J 16: 544-549.

14. Werneburg GT, Kongnyuy M, Halpern DM, et al. (2018) Effects of focal vs total cryotherapy and minimum tumor temperature on patient-reported quality of life compared with active surveillance in patients with Prostate Cancer. Urology 113: 110-118.

15. Habibian DJ, Katz AE (2016) Emerging minimally invasive procedures for focal treatment of organ-confined prostate cancer. Int J Hyperthermia 32: 795-800.

16. Klotz $L$ (2020) Active surveillance in intermediate-risk prostate cancer. BJU Int 125: 346-354.

17. Romero-Otero J, García-Gómez B, Duarte-Ojeda JM, et al. (2016) Active surveillance for prostate cancer. Int J Urol 23: 211-218.

18. Witherspoon L, Breau RH, Lavallée LT (2020) Evidence-based approach to active surveillance of prostate cancer. World J Uro 38: 555-562.

19. Newcomb LF, Thompson IM, Boyer HD, et al. (2016) Outcomes of active surveillance for clinically localized prostate cancer in the prospective, multi-institutional canary pass cohort. J Urol 195: $313-320$

20. (2019) Overview | Prostate cancer: Diagnosis and management | Guidance | NICE.

21. Morash C, Tey R, Agbassi C, et al. (2015) Active surveillance for the management of localized prostate cancer: Guideline recommendations. Can Urol Assoc J 9: 171-178.

22. Sanda MG, Cadeddu JA, Kirkby E, et al. (2018) Clinically localized prostate cancer: AUA/ASTRO/SUO guideline. Part I: Risk stratification, shared decision making, and care options. J Urol 199: 683-690.

23. Mottet N, Bellmunt J, Bolla M, et al. (2017) EAU-ESTRO-SIOG Guidelines on prostate cancer. Part 1: screening, diagnosis, and local treatment with curative intent. Eur Urol 71: 618-629.

24. Tosoian JJ, Mamawala M, Epstein JI, et al. (2015) Intermediate and longer-term outcomes from a prospective activesurveillance program for favorable-risk prostate cancer. J Clin Oncol 33: 3379-3385.

25. Bul M, Zhu X, Valdagni R, et al. (2013) Active surveillance for lowrisk prostate cancer worldwide: The PRIAS study. Eur Urol 63: 597-603.

26. Adamy A, Yee DS, Matsushita K, et al. (2011) Role of prostate specific antigen and immediate confirmatory biopsy in predicting progression during active surveillance for low-risk prostate cancer. J Urol 185: 477-482.

27. Klotz L, Vesprini D, Sethukavalan P, et al. (2015) Long-term follow-up of a large active surveillance cohort of patients with prostate cancer. J Clin Oncol 33: 272-277.

28. Loeb S, Zhou Q, Siebert U, et al. (2017) Active surveillance versus watchful waiting for localized prostate cancer: A model to inform decisions. Eur Urol 72: 899-907.

29. Wei JT, Dunn RL, Litwin MS (2000) Development and validation of the expanded prostate cancer index composite (EPIC) for comprehensive assessment of health-related quality of life in men with prostate cancer. Urology 56: 899-905.

30. Rosen RC, Riley A, Wagner G, et al. (1997) The international index of erectile function (IIEF): A multidimensional scale for assessment of erectile dysfunction. Urology 49: 822-830.

31. Marra G, Soeterik T, Oreggia D, et al. (2021) Long-term outcomes of focal cryotherapy for low- to intermediate-risk prostate cancer: Results and matched pair analysis with active surveillance. Eur Urol Focus

32. Zhou JT, Fang DM, Xia S, et al. (2019) The incidence proportion of erectile dysfunction in patients treated with cryotherapy for prostate cancer: A meta-analysis. Clin Transl Oncol 21: 11521158.

33. Litwin MS, Hays RD, Fink A, et al. (1995) Quality-of-life outcomes in men treated for localized prostate cancer. JAMA 273: 129-135.

34. Robinson JW, Donnelly BJ, Saliken JC, et al. (2002) Quality of life and sexuality of men with prostate cancer 3 years after cryosurgery. Urology 60: 12-18.

35. Avulova S, Zhao Z, Lee D, et al. (2018) The effect of nerve sparing status on sexual and urinary function: 3-year results from the ceasar study. J Urol 199: 1202-1209.

36. Hoffman KE, Penson DF, Zhao Z, et al. (2020) Patient-reported outcomes through 5 years for active surveillance, surgery, brachytherapy, or external beam radiation with or without androgen deprivation therapy for localized prostate cancer. JAMA 323: 149-163.

37. Han KR, Cohen JK, Miller RJ, et al. (2003) Treatment of organ confined prostate cancer with third generation cryosurgery: Preliminary multicenter experience. J Urol 170: 1126-1130.

38. Onik G, Narayan P, Vaughn D, et al. (2002) Focal "nerve-sparing" cryosurgery for treatment of primary prostate cancer: A new approach to preserving potency. Urology 60: 109-114.

39. Tay KJ, Polascik TJ (2016) Focal cryotherapy for localized prostate cancer. Arch Esp Urol 69: 317-326.

40. Mendez MH, Passoni NM, Pow-Sang J, et al. (2015) Comparison of outcomes between preoperatively potent men treated with focal versus whole gland cryotherapy in a matched population. J Endourol 29: 1193-1198.

41. Shah TT, Peters M, Miah S, et al. (2021) Assessment of return 
Citation: Mendelson J, Sommer J, Akerman M, et al. (2021) Bowel, Urinary, and Sexual Function in Patients with Low to Intermediate Risk Prostate Cancer on Active Surveillance and Primary Focal Cryotherapy. Ann Minim Invasive Surg 3(1):24-30

to baseline urinary and sexual function following primary focal cryotherapy for nonmetastatic prostate cancer. Eur Urol Focus 7: 301-308

42. Lardas M, Liew M, Van Den Bergh RC, et al. (2017) Quality of life outcomes after primary treatment for clinically localised prostate cancer: A systematic review. Eur Urol 72: 869-885.
43. Werneburg GT, Kongnyuy M, Halpern DM, et al. (2018) Patientreported quality of life progression in men with prostate cancer following primary cryotherapy, cyberknife, or active holistic surveillance. Prostate Cancer Prostatic Dis 21: 355-363.

DOI: $10.36959 / 351 / 587$

Copyright: (C) 2021 Mendelson J, et al. This is an open-access article distributed under the terms of the Creative Commons Attribution License, which permits unrestricted use, distribution, and reproduction in any medium, provided the original author and source are credited. 